\title{
Impact of alloyed capping layers on the performance of InAs quantum dot solar cells
}

\author{
A.D. Utrilla , J.M. Ulloa ， Ž. Gačević , D.F. Reyes ，I. Artacho , T. Ben ，D. González ， \\ A. Hierro , A. Guzman
}

\begin{abstract}
A B S T R A C T
The impact of using thin GaAs(Sb)(N) capping layers (CLs) on InAs/GaAs quantum dots (QDs) is investigated for their application in solar cell devices. We demonstrate the ability to combine strain-balancing techniques with band engineering approaches through the application of such CLs. Extended photoresponse is attainable by means of an independent tunability of the electron and hole confinements in the $\mathrm{QD}$. Moreover, the CL acts itself as a quantum well (QW), providing an additional photoresponse, so that the devices work as hybrid QD-QW solar cells. The use of a GaAsSb CL is particularly beneficial, providing devices with efficiencies under AM1.5 conditions 20\% higher than standard GaAs-capped QDs. This is mainly due to a significant increase in photocurrent beyond the GaAs bandgap, leading to an enhanced short-circuit current density $\left(J_{s c}\right)$. The addition of $\mathrm{N}$ to the CLs, however, produces a strong reduction in $J_{s c}$. This is found to be related to carrier collection problems, namely, hindered electron extraction and retrapping in the CLs. Nevertheless, the application of reverse biases induces a release of the trapped carriers assisted by a sequential tunneling mechanism. In the case of GaAsN CLs, this leads to a complete carrier collection and reveals an even higher QD-QW-related photocurrent than when using a GaAsSb CL. The hindered carrier collection is stronger in the case of the quaternary CLs, likely due to the faster recombination rates in the type-I GaAsSbN/GaAs QW structure as compared to the type-II ternary counterparts. Nevertheless, alternative approaches, such as the use of a thinner CL or a short-period superlattice CL, lead to significant improvements, demonstrating a great potential for the quaternary CLs under a proper device design.
\end{abstract}

\section{Introduction}

Recently, quantum dots (QDs) have been considered as a feasible opportunity to exceed the Shockley-Queisser efficiency limit of single-gap materials [1]. On one side, electron-hole pair multiplication, predicted to provide higher conversion efficiency limits [2], is expected to become a competitive mechanism in zerodimensional structures [3]. Indeed, efficiencies in QD-based solar cells exhibiting multiple-exciton generation have been theorized to undergo significant increases as compared to bulk material and quantum efficiencies higher than 1 have already been demonstrated $[4,5]$. On the other side, the use of QDs has also been proposed as a possibility to realize the intermediate-band solar cell concept [6]. Particularly, the use of the well-known InAs/GaAs system has been the focus of increasing efforts to take advantage of these approaches and implement QD-based enhanced solar cells. The introduction of absorption levels by the QDs leads to an extended photoresponse beyond the GaAs band edge, allowing the achievement of higher short-circuit currents [7-10]. Further control of the absorption QD energy levels can be obtained through the use of a modified thin capping layer (CL) on top of the QDs, which could allow a precise control and extension of the absorption energy. Moreover, the CL could also act itself as a quantum well (QW), providing an additional photoresponse at energies below the GaAs band edge. However, although improved solar cell characteristics could be expected, this approach has been all but unexplored in this field $[9,11]$. 
Particularly, the application of a thin GaAsSbN CL could be of great interest on this point, allowing engineering the band structure as well as the strain of the QDs. On one side, the beneficial impact of thin GaAsSb CLs on InAs QDs properties is already well known [12], where the presence of Sb extends the QD ground state wavelength by reducing the valence band offset [13]. The use of such a CL has been found to significantly enhance the efficiency of both light emitting devices [14,15] and photodetectors [16], while improved solar cell characteristics have been also recently reported in stacked-QD devices [9]. However, a limitation may be found regarding the number of layers that can be stacked due to strain accumulation, since GaAsSb CLs increase the overall compressive strain in the stack. On the other side, GaAsN CLs have also been found to improve the InAs QD luminescence for low $\mathrm{N}$ contents $[17,18]$. With this $\mathrm{CL}$, the emission wavelength is extended because of the QD-CL conduction band offset reduction induced by the presence of $\mathrm{N}$ [19]. Furthermore, the presence of $\mathrm{N}$ reduces the accumulated strain, allowing the stacking of a large number of QD layers [20]. Thus, the combination of both elements, Sb and $\mathrm{N}$, could take advantage of both approaches. The use of $\mathrm{GaAs}(\mathrm{Sb})$ (N) CLs would allow an individual control of carrier escape times through the possibility to tune both the electron and hole confinements [21]. In addition, a suitable ratio of $\mathrm{Sb}$ to $\mathrm{N}$ in the $\mathrm{CL}$ would compensate the accumulated strain, allowing a higher number of stacked QD layers before relaxation via the origination of extended defects. This would also be expected to mitigate the loss in open-circuit voltage $\left(V_{o c}\right)$ induced by the presence of InAs QDs, as found through different strain-balancing techniques [2224].

In this work we perform a detailed comparative study concerning the impact of thin $\mathrm{GaAs}(\mathrm{Sb})(\mathrm{N})$ CLs on the overall performance of InAs/GaAs QD-based p-i-n junction solar cells. We will show how the presence of a CL plays a major role in the photoresponse, providing an additional contribution to the photocurrent (PC). However, a problem arises regarding carrier collection, intrinsically related to the introduction of $\mathrm{N}$, so that $\mathrm{N}$-containing CLs act as potential wells deep enough to hinder carrier transport and extraction and degrade the final characteristics of the device. This drawback is systematically addressed, from both experimental and theoretical points of view, and new possible approaches to circumvent this undesired effect are proposed.

\section{Experimental details}

All of the $\mathrm{p}-\mathrm{i}-\mathrm{n}$ junction solar cells under study were grown by solid-source molecular beam epitaxy on $n^{+}$-GaAs (001) substrates under $\mathrm{As}_{4}$-stabilized conditions. Due to the comparative nature of this study, a simple device structure was chosen so no window layer or antireflection coating was used (parameters such as the doping levels or the emitter and intrinsic region thicknesses are not either optimized). A schematic diagram of the structure is shown in Fig. 1. A 500 nm-thick n-GaAs base buffer was grown at $580^{\circ} \mathrm{C}$, while the emitter consists of $350 \mathrm{~nm}$-thick p-GaAs followed by a $150 \mathrm{~nm}$-thick $\mathrm{p}^{+}$-GaAs. The active region always consists of $10 \mathrm{QD}$ layers embedded in the middle of $400 \mathrm{~nm}$-thick intrinsic GaAs. QDs were grown by depositing 2.8 monolayers (MLs) of InAs at $450{ }^{\circ} \mathrm{C}$ and $0.04 \mathrm{ML} \mathrm{s}^{-1}$ resulting in a density, estimated through atomic force microscopy scans of similar uncapped QDs, near $3 \cdot 10^{10} \mathrm{~cm}^{-2}$. The different $\mathrm{CL}$ materials, described below, were always deposited at $470^{\circ} \mathrm{C}$, immediately followed by a $2.5 \mathrm{~nm}$-thick low-temperature (LT) GaAs layer to avoid $\mathrm{Sb} / \mathrm{N}$ desorption. Subsequently, $30 \mathrm{~nm}$-thick GaAs spacers were grown at high temperature $\left(580^{\circ} \mathrm{C}\right)$, found to be a critical factor for solar cell characteristics [25].

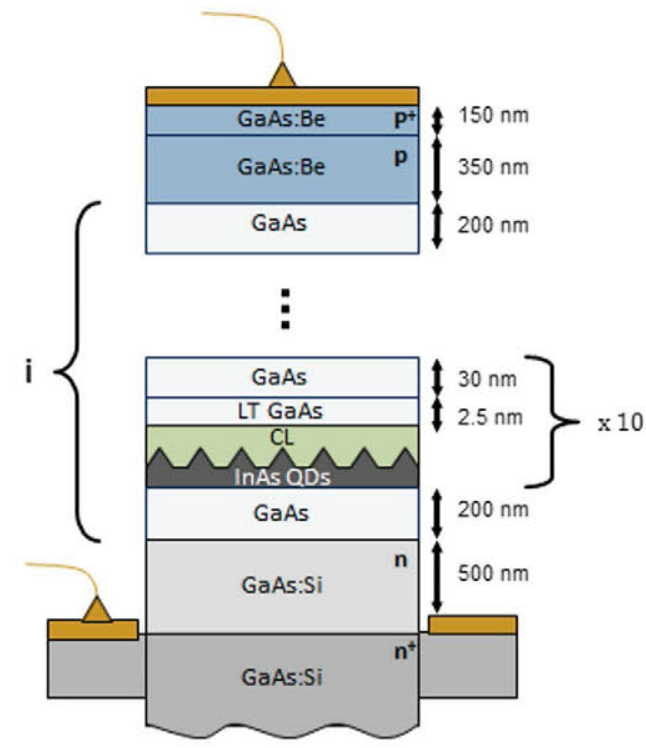

Fig. 1. Schematic sketch of the $\mathrm{p}-\mathrm{i}-\mathrm{n}$ devices containing 10 stacked InAs QD layers capped with a thin $\mathrm{GaAs}(\mathrm{Sb})(\mathrm{N}) \mathrm{CL}$.

To get a detailed insight into the structural properties, conventional transmission electron microscopy (TEM) analyses were performed using a JEOL JEM-2100 LaB6 operating at $200 \mathrm{kV}$. The structural analysis was complemented with X-ray diffraction (XRD). Unlike TEM, the XRD is a rapid and a non-destructive technique which retrieves structural information averaged over extended sample area $\left(>1 \mathrm{~mm}^{2}\right)$. The measurements were performed with $C u$-Ko 1 line $\left(\lambda_{\mathrm{x}}=1.5406 \AA\right)$, in a commercial Panalytical X'Pert Pro diffractometer equipped with a $\mathrm{Ge}(220)$ hybrid monocromator.

Photoluminescence (PL) measurements were performed using a He-Ne laser as excitation source. The emitted light was collected, dispersed through a $1 \mathrm{~m}$-spectrometer, and then detected with a nitrogen-cooled Ge detector.

The test devices consisted of $200 \mu \mathrm{m}$-diameter cylindrical mesas processed by standard fabrication techniques, with AuGe/ $\mathrm{Au}$ and $\mathrm{Au} / \mathrm{AuZn} / \mathrm{Au}$ for the $\mathrm{n}$ - and p-type contacts, respectively. Photocurrent density-voltage $(J-V)$ measurements were conducted using a Keithley 2400 sourcemeter. The light source is a solar simulator equipped with a $150 \mathrm{~W}$ Xenon Lamp and an AM $1.5 \mathrm{D}$ filter combination (Newport). The total light intensity was calibrated to $900 \mathrm{~mW} / \mathrm{cm}^{2}$ using a GaAs reference cell. PC and $I-V$ measurements under monochromatic illumination were carried out using a Keithley 230 voltage source and a Keithley 617 electrometer, utilizing light from a quartz halogen lamp dispersed through a $0.34 \mathrm{~m}$-monochromator.

\section{Results and discussions}

The comparative analysis was carried out using $5 \mathrm{~nm}$-thick GaAsSbN, GaAsSb, and GaAsN CLs (samples S-SbN, S-Sb, and S-N, respectively). A $2 \mathrm{ML} \mathrm{s}^{-1}$ growth rate, particularly beneficial for $\mathrm{N}$-containing samples [26], was used for the growth of the $\mathrm{CL}$ in all these samples. The $\mathrm{Sb}$ and $\mathrm{N}$ nominal contents were always set to $10 \%$ and $2.0 \%$, respectively. In addition, a sample containing GaAscapped QDs (sample S-0) and a control GaAs sample, with no QDs inside, were grown for comparison. 

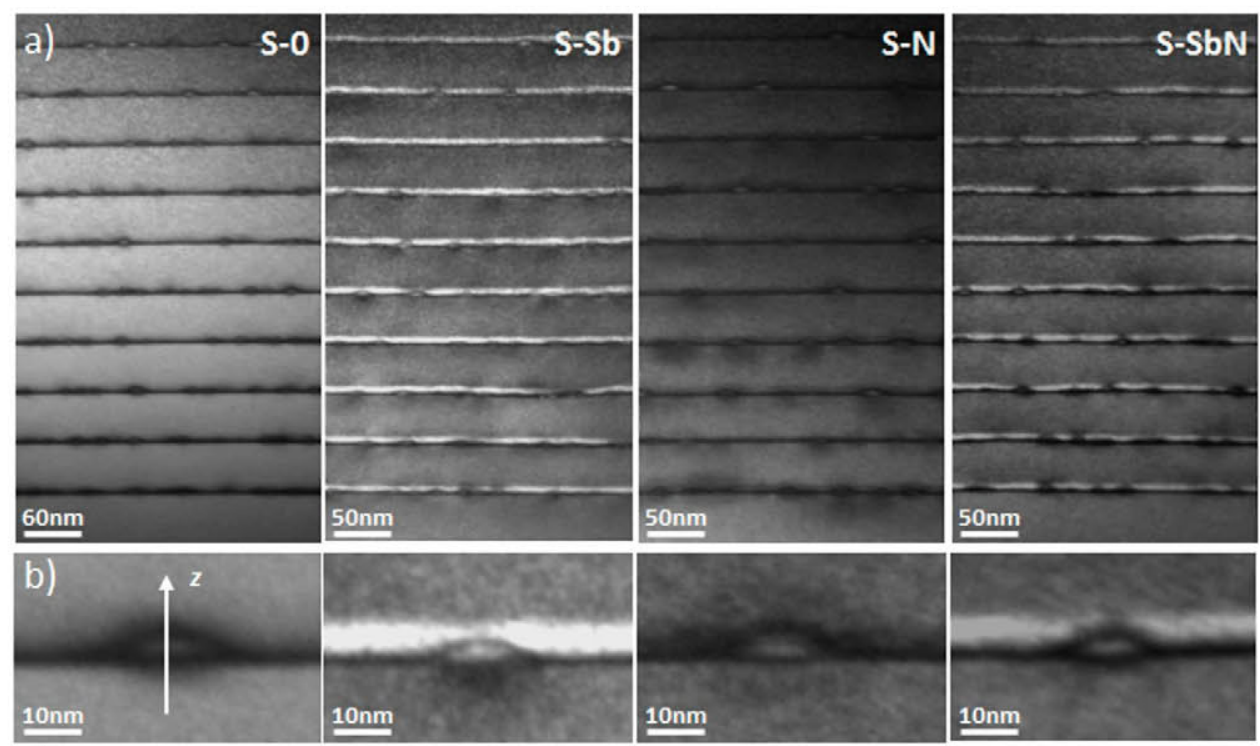

c)
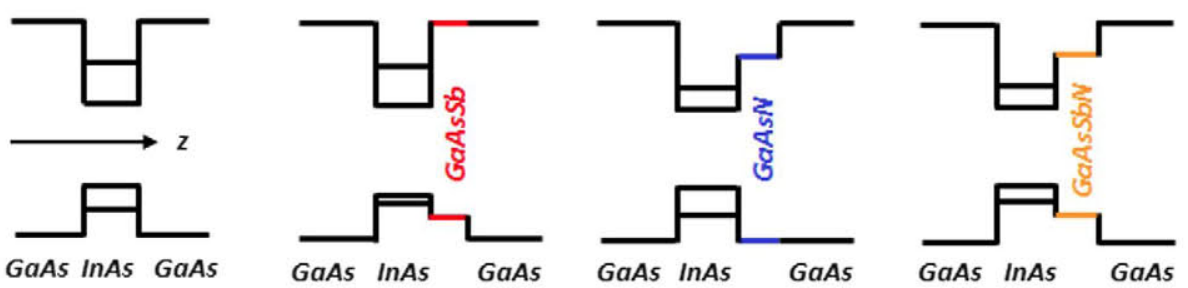

Fig. 2. (a) Dark field 002 TEM image from samples S-0, S-Sb, S-N, and S-SbN, from left to right. Zoom of one representative QD (b) and the sketch of the expected band structure in each sample (c).

\subsection{Structural analysis and photoluminescence}

Chemically sensitive dark field 002 TEM images in Fig. 2 (a) show global view of the 10 QD layers of samples S-0, S-Sb, S-N, and S-SbN (from left to right, respectively). A representative $\mathrm{QD}$, from each sample, has been zoomed in for more detail (Fig. 2(b)). No extended defects were found in any of the studied samples, even for the case of $\mathrm{S}-\mathrm{Sb}$, expected to accumulate the highest strain. The growth front remains flat all through the structure in each sample. All of them present a period of approximately $40 \mathrm{~nm}$, close to the expected value, while that of the reference sample is around $50 \mathrm{~nm}$, as a consequence of a deviation from the nominal GaAs growth rate during the growth. The CLs can be well distinguished: the presence of $\mathrm{N}$ results in a darker contrast in sample S-N, while Sb yields a brighter contrast in both Sb-containing samples. The CL-GaAs interface appears well defined, with the $\mathrm{CL}$ covering homogeneously the QDs in all cases. This indicates that composition modulation in the $\mathrm{CL}$ is suppressed to high extent even in the case of the quaternary alloy, likely due to the beneficial impact of the high growth rates $[26,27]$. The CL thickness remains virtually constant in all samples and was estimated to be approximately $5.5 \mathrm{~nm}$, near the nominal thickness. For the sake of clarity, a sketch of the expected QD-CL band structure for a single layer in each sample is represented in Fig. 2(c).

Fig. 3(a) shows high resolution XRD $\theta-2 \theta$ scans around the (004) reflection as a function of the CL material. The presence of abrupt heterointerfaces with an exceptionally high periodicity throughout the superlattice structure results in the clearly observed satellite peaks. The superlattice period thickness can be more precisely calculated from the Bragg angular position of two successive satellites, $S(n)$ and $S(n+1)$, considering the X-ray wavelength. Period thicknesses of $48,40,41$, and $40 \mathrm{~nm}$ are obtained for $\mathrm{S}-0, \mathrm{~S}-\mathrm{Sb}, \mathrm{S}-\mathrm{N}$, and $\mathrm{S}-\mathrm{SbN}$, respectively, in good agreement with those estimated from TEM images. The shift of the

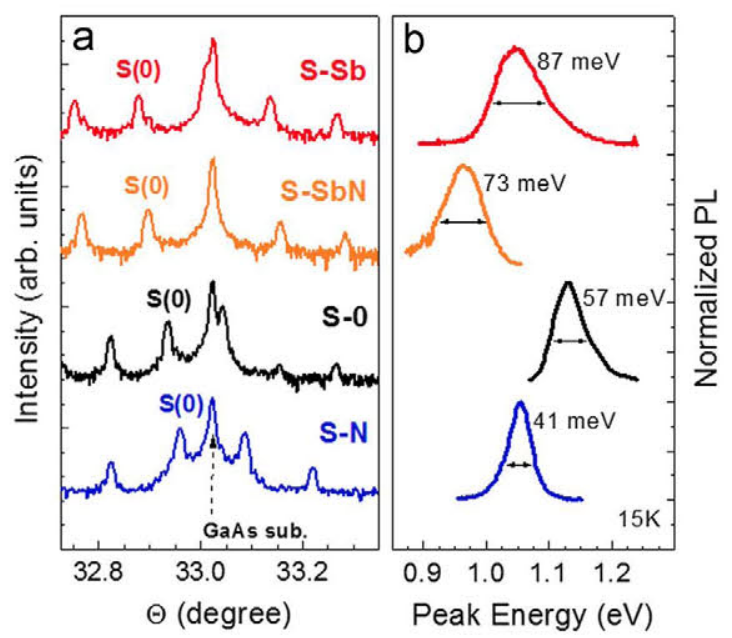

Fig. 3. $\theta / 2 \theta$ XRD scans around 004 Bragg reflection performed on samples S-0, S-Sb $\mathrm{S}-\mathrm{N}$, and $\mathrm{S}-\mathrm{SbN}$ (left), showing that the increasing strain is directly correlated with the PL FWHM (right).

superlattice mean peak $(S(0))$ to a lower angle with respect to the GaAs substrate Bragg angle in sample $\mathrm{S}-0$ is a fingerprint of compressive strain, which has been induced by the InAs QD insertion into the GaAs matrix. The average strain is partially compensated by applying a GaAsN CL, as the smaller shift of the zeroth order satellite peak indicates. Thus, the GaAsN CL is acting as a strain compensating layer $[20,28]$. Also, as observed in Fig. 3 (a), sample S-Sb presents the highest accumulated strain, which is relatively compensated by adding $\mathrm{N}$ together with $\mathrm{Sb}$ in sample $\mathrm{S}-\mathrm{SbN}$. However, there is still an accumulation of compressive strain with respect to sample $\mathrm{S}-0$, due to the fact that the $\mathrm{Sb} / \mathrm{N}$ 
ratio $(\approx 10 / 2)$ is higher than that for lattice-matching condition $(\approx 10 / 4)$ [29]. The normalized PL spectra from this series of samples (Fig. 3(b)), depicted together to each respective rocking curve, reveal a direct correlation between the PL full width at halfmaximum (FWHM) and the accumulated strain. The PL FWHM increases monotonically with the accumulated strain, regardless of the $\mathrm{CL}$ material and emission wavelength. This tendency is not followed in single QD-layer samples, wherein similar amounts of $\mathrm{Sb}$ in GaAsSb CLs induce a reduction of the FWHM [12]. This implies that strain becomes the dominant factor affecting the PL linewidth for 10 stacked QD layers. Regarding the PL peak energies, the shifts with respect to the reference sample are 64,93 , and $163 \mathrm{meV}$ for S-N, S-Sb, and S-SbN, respectively. The energy shift for sample $\mathrm{S}-\mathrm{SbN}$ is therefore approximately the same that the one that results from the addition of those in samples S-Sb and S-N. This confirms that the effect of $\mathrm{Sb}$ and $\mathrm{N}$ on the band structure are cumulative (see Fig. 2(c)), and, therefore, the electron and hole confinements can be independently tuned in stacked QD layers.

\subsection{Solar cell performance}

Fig. 4 shows the $I-V$ characteristics under 1 sun illumination for samples S-0, S-Sb, and S-SbN, together with that of the control sample. The control sample shows a low short-circuit current density $\left(J_{s c}\right)$ as compared to common values for GaAs solar cells, which is due to the unoptimized design of the solar cell previously mentioned above. The introduction of InAs QDs (S-0) leads to an expected reduction of the $V_{\mathrm{oc}}$, in this case of $0.145 \mathrm{~V}$. However, also a drop is observed as to its $J_{s c}$. A possible reason for this will be described in Section 3.7. In the case of sample S-Sb, $V_{\mathrm{oc}}$ is slightly reduced in $0.022 \mathrm{~V}$ with respect to that of S-0, while $J_{s c}$ is significantly increased, leading to an efficiency, $20 \%$ higher than that of sample S-0. Regarding both these samples, the presence of some parasitic resistances, being responsible for losses in $V_{o c}$ and $J_{S c}$, could be in principle expected from the shape of their $I-V$ curves.

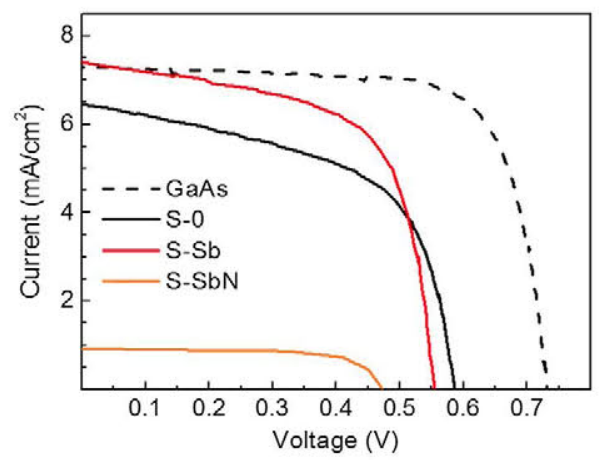

Fig. 4. Current-voltage characteristics of samples S-0, S-Sb, and S-SbN, together with that of the control sample, under $900 \mathrm{~W} / \mathrm{m}^{2}$ AM1.5 conditions.

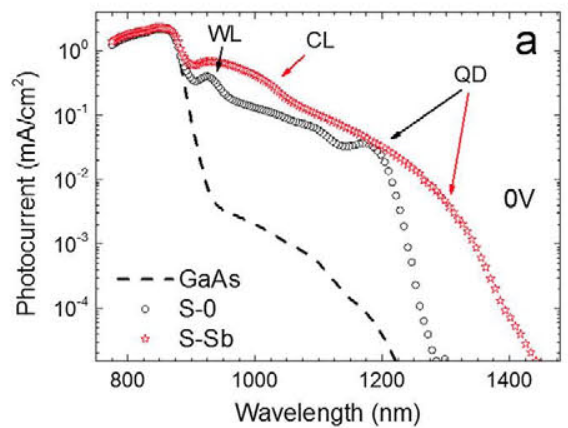

On the other side, the addition of $\mathrm{N}$ leads to a drastic reduction in $J_{s c}$, despite the expected benefits from using simultaneously $\mathrm{Sb}$ and $\mathrm{N}$ in the $\mathrm{CL}$ (among them, the $\mathrm{N}$-related reduction of the introduced strain). $V_{o c}$ is also further reduced in $\mathrm{S}-\mathrm{SbN}$, so that it seems to be restricted as a function of the thin CL ground state energy. Nevertheless, the electron escape rate could be also having a relevant impact on $V_{\text {oc }}$ [30], as it will be discussed later. Remarkably, the best performance is found in the sample with the highest accumulated strain (and largest PL FWHM), indicating that other factors are more critical in this case.

\subsection{Photocurrent spectra}

RT PC from samples $\mathrm{S}-0, \mathrm{~S}-\mathrm{Sb}$, and the control sample are shown in Fig. 5(a). The introduction of InAs QD layers embedded in the GaAs structure ( $\mathrm{S}-0$ ) extends the PC to longer wavelengths, as compared to the control sample, due to the well-identified contribution of the QDs and the wetting layer (WL). The PC originated from GaAs over the measured range of wavelengths is slightly reduced by the introduction of InAs QDs (this will be analyzed in detail in Section 3.7). This should in principle be on the origin of the reduced $J_{\mathrm{sc}}$ compared to the control sample. Regarding sample $\mathrm{S}-\mathrm{Sb}$, the photoresponse is now further extended to longer wavelengths, as a consequence of the modified QD ground state. $\mathrm{PC}$ from the ground state shows now a broader absorption edge than in case of $\mathrm{S}-0$, as expected from the PL FWHM. In this sample, the presence of the CL significantly contributes to the PC, acting as a type-II GaAsSb/GaAs QW [13], and therefore the device works as a hybrid QD-QW solar cell. In addition, the GaAs contribution to the $P C$ over the measured range remains unaltered. This, together with the increase in $J_{s c}$, means that the presence of the GaAsSb QW, which actually creates a small barrier for electrons and a potential well for holes [13], seems to have no significant impact on the transport of carriers generated at the GaAs layers. On the contrary, the additional photogenerated current is now contributing to an increase in $J_{\mathrm{Sc}}$.

RT PC from samples $\mathrm{S}-\mathrm{N}$ and $\mathrm{S}-\mathrm{SbN}$ are shown in Fig. 5 (b) together with that of sample $\mathrm{S}-0$ for a comparison. The use of a GaAsN CL extends the photoresponse beyond that of sample S-0, where the contribution of the QD ground state and the $\mathrm{CL}$, acting as a type-II GaAsN/GaAs QW [31], is clearly observed. However, PC originating from the GaAs layers is now significantly reduced. This is also observed in sample S-SbN, in which there is also a weaker contribution of the QD-CL structure to the PC. In spite of the fact that $\mathrm{N}$ is reducing the accumulated strain, the addition of $\mathrm{N}$ yields a degradation of the GaAs contribution to the total PC, giving rise to the observed drastic fall of $J_{\mathrm{sc}}$.

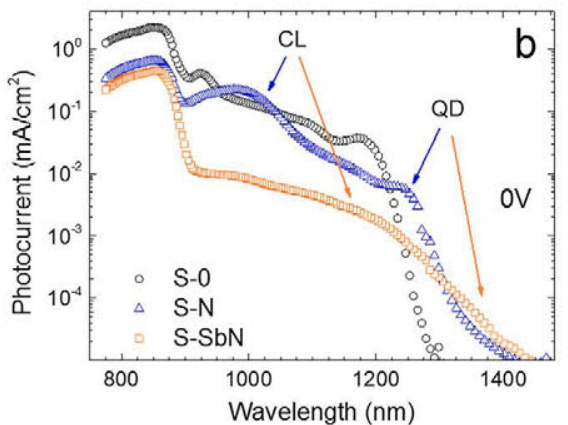

Fig. 5. (a) RT PC spectra from the control GaAs sample, S-0, and S-Sb. (b) RT PC spectra from samples S-N and S-SbN together with that from S-0 as reference. 

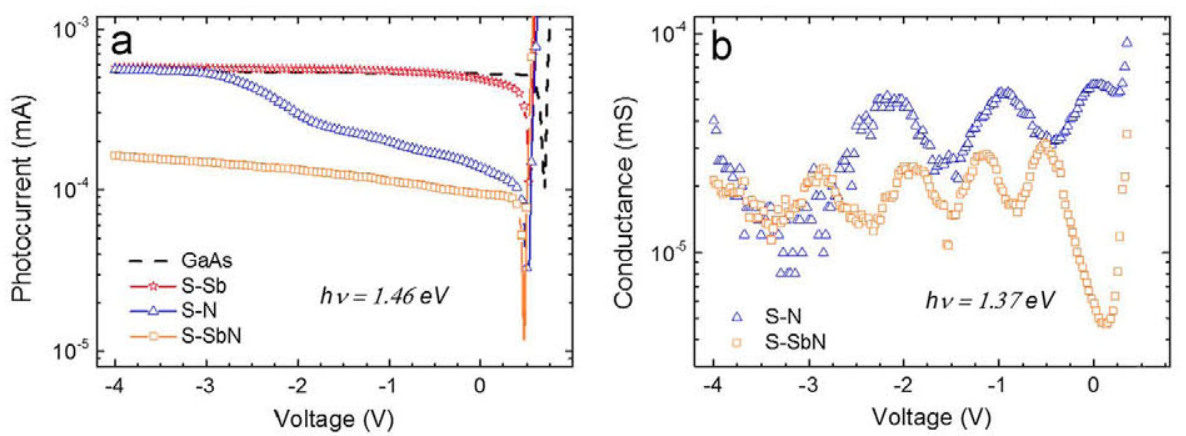

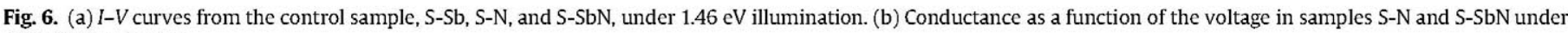
$1.37 \mathrm{eV}$ illumination.

\subsection{I-V measurements under illumination: effect of the $N$ incor- poration on carrier collection}

In order to understand the mechanisms involved in the previously discussed reduction of the PC and $J_{s c}$ in $\mathrm{N}$-containing samples, $I-V$ measurements under monochromatic illumination were carried out in samples S-Sb, S-N, and S-SbN. First, $I-V$ curves were taken under $1.46 \mathrm{eV}$ illumination, over the GaAs bandgap energy, and represented together with that of the control sample (Fig. 6(a)). Reverse biases up to $-4 \mathrm{~V}$ were applied. An increase of the photogenerated current with the applied reverse bias indicates an incomplete carrier collection [32]. Hence, the nearly constant value of the PC with voltage in sample S-Sb confirms that the GaAsSb CLs are hardly affecting carrier transport, but for low biases. The clear tendency to a saturation current also indicates that no leakage shunt resistance is responsible for the enhanced slope of the current fall in the AM1.5 $I-V$ curve, since a low shunt resistance should lead to an indefinite increase of the reverse current. The situation is completely different in $\mathrm{N}$-containing samples, where a strong dependence of the current on the applied bias is observed. Despite being much lower at $0 \mathrm{~V}$, the PC from sample S-N merges with that from S-Sb and the control sample at $-4 \mathrm{~V}$. This points to the fact that the GaAsN CLs are significantly limiting the extraction of the photogenerated carriers at low biases, likely as a result of an electron-trapping mechanism. In the case of sample S-SbN, increasing the reverse bias does not yield a total PC comparable to that from the other three samples. Hence, some additional carrier loss mechanism must be present, such as recombination at point defects that may be generated by the simultaneous presence of $\mathrm{Sb}$ and $\mathrm{N}$. In addition, the type-I band structure in the GaAsSbN/GaAs QWs implies a faster radiative recombination than in the type-II GaAsSb and GaAsN QWs, which could also explain the degraded carrier extraction efficiency observed in this sample. Higher built-in fields would allow a more efficient extraction in samples where carrier collection is incomplete, increasing PC and thereby $V_{\text {oc }}$ [33]. This is feasible by reducing the thickness of the intrinsic GaAs layer and/or the spacer (without reaching the coupling limit).

Illuminating with light below the GaAs bandgap energy, most carriers are generated inside the QWs. Under such conditions, a possible hindered extraction of the photogenerated carriers from the QWs is added to the retrapping effects. Indeed, the conductance in samples S-N and S-SbN (Fig. 6(b)), calculated as the first derivative of their $I-V$ curves, shows an oscillatory behavior with the applied bias, not present in sample S-Sb. This reveals the presence of a resonant Fowler-Nordheim tunneling component, resulting from an accumulation of negative charge in the QWs $[34,35]$. This is caused, in its turn, by a significant difference between the electron and hole escape times, whereby holes escape much faster so that electrons cannot recombine. Indeed,

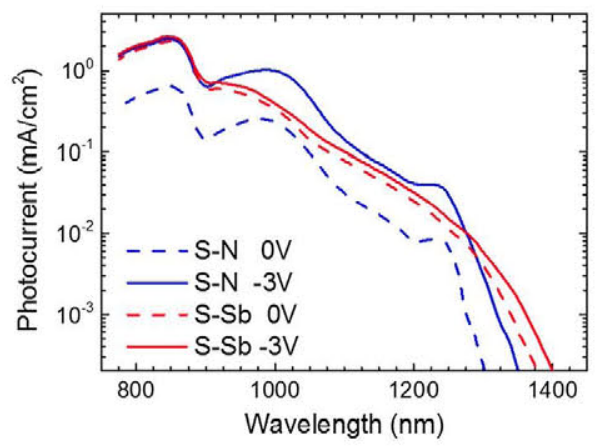

Fig. 7. RT PC spectra from samples $\mathrm{S}-\mathrm{N}$ and $\mathrm{S}-\mathrm{Sb}$ at $0 \mathrm{~V}$ and $-3 \mathrm{~V}$ bias.

the electron accumulation would locally screen the built-in field, contributing to explain the sharp drop in $V_{\mathrm{oc}}$ [30]. In addition, this accumulation of negative charge suggests also a presumed accumulation inside the QD, what could also be increasing Coulombian scattering through a higher density of confined carriers [36].

Fig. 7 shows the PC spectra at $0 \mathrm{~V}$ and $-3 \mathrm{~V}$ from samples $\mathrm{S}-\mathrm{Sb}$ and S-N. PC from sample S-Sb shows a slight increase over the whole spectrum range when applying the reverse bias, while that from sample S-N undergoes such a strong increase that PC originating from GaAs is now as high as that in S-Sb sample. Remarkably, the contribution of the QD-CL structure is now significantly higher than that of S-Sb, contrary to what happens at $0 \mathrm{~V}$. Two conclusions can be drawn from these results. Firstly, the QD-CL system in sample $\mathrm{S}-\mathrm{N}$ is absorbing more efficiently than in sample $\mathrm{S}-\mathrm{Sb}$, but carriers cannot be extracted except for high reverse applied bias. Secondly, the extraction of photogenerated carriers is hindered by retrapping in the $\mathrm{N}$-containing QWs. This means that the reduced $\mathrm{PC}$ at $0 \mathrm{~V}$ in $\mathrm{S}-\mathrm{N}$ is not due to the presence of $\mathrm{N}$-induced point defects. Moreover, this would now fit with a positive impact of strain reduction in the structure, which was hidden by reduced collection efficiency at $0 \mathrm{~V}$. Hence, it may be possible to further exploit such a structure through the optimization of the built-in electric field for a more efficient extraction at operation voltages.

\subsection{Carrier escape times: theoretical modelling}

The reduced carrier extraction efficiency in sample S-N should be in principle related to long electron escape times from the GaAsN QWs, while the hole escape time from GaAsSb QWs should be much faster. In order to theoretically assess the carrier escape times from such CLs, the energy levels in these QWs were calculated solving a one-dimensional Schrödinger equation through a finite-difference method. With the purpose of simplifying and due to the fact that no precise description is available for every sample, 
no WL was included in the simulations. The thickness of the QWS was taken to be $5.5 \mathrm{~nm}$, as found by TEM, whereas the nominal Sb and $\mathrm{N}$ contents, i.e., $10 \%$ and $2 \%$, respectively, were used. Regarding the GaAsSb QWs, the bandgap energy and band offsets were estimated at RT from experimental results for GaAsSb pseudomorphically grown on GaAs [13], while the hole effective mass is obtained from a linear interpolation between the binaries. As for the GaAsN QWs, the bandgap energy and band offsets were considered as predicted by the band anticrossing model (BAC) in first order perturbation theory [19], with the specific values for the electron effective mass and BAC parameters as described in Ref. [37]. The results from the simulations are depicted in Fig. 8. Three confined levels were found for the GaAsSb QWs, with an escape barrier of $164 \mathrm{meV}$ for the ground state. For the case of GaAsN QWs, two energy levels were obtained, the barrier for the ground state being $291 \mathrm{meV}$. Once the confined energy levels are known for the QWs, the thermal carrier escape times $\tau_{\mathrm{th}}$ can be estimated, given by [38]:

$\frac{1}{\tau_{\mathrm{th}}}=\frac{1}{L_{\mathrm{w}}} \sqrt{\frac{k_{\mathrm{B}} T}{2 \pi m^{*}}} \exp \left(-\frac{\Delta E}{k_{\mathrm{B}} T}\right)$

where $L_{\mathrm{w}}$ is the width of the QW, $m^{*}$ is the carrier effective mass inside the confined structure, $k_{\mathrm{B}}$ is Boltzmann constant, $T$ is temperature, and $\triangle E$ is the barrier height. With this expression, the RT thermal escape time from the ground state for holes in the GaAsSb QW and electrons in the GaAsN QW are estimated to be approximately 0.08 and $6 \mathrm{~ns}$, respectively. Thus, the electron escape time is around two orders of magnitude longer than that of holes, being so high that radiative recombination may become a competitive mechanism, despite the type-II band alignment. This is in agreement with the experimental results in the previous section, supporting the hypothesis that electron trapping and hindered

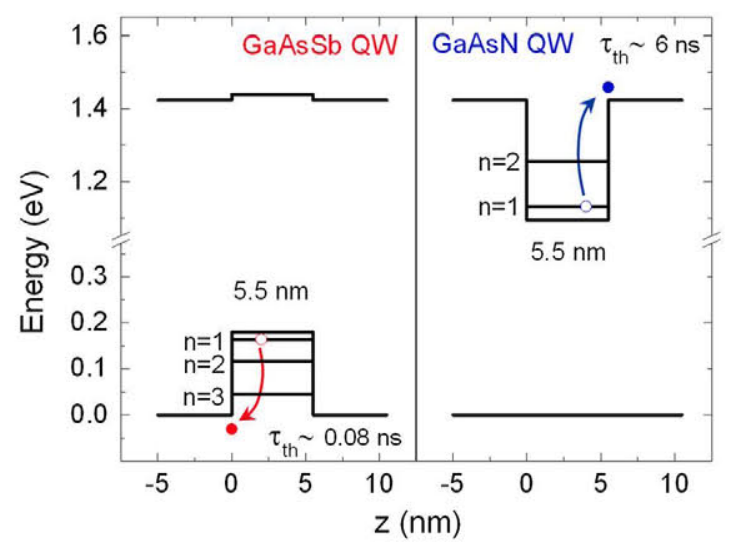

Fig. 8. Results from finite-differences method simulations for confined energy states and carrier escape times in GaAsSb/GaAs and GaAsN/GaAs QWs. extraction are responsible for the carrier collection losses in the $\mathrm{N}$-containing samples. Under no applied field, tunneling escape is negligible and the high electron escape time leads to an accumulation of negative charge giving rise to the formation of highfield domains in the N-containing QWs [35]. This leads to a sequential Fowler-Nordheim tunneling mechanism with the application of a reverse bias, which is superimposed to the thermal escape component and is responsible for the observed oscillations in the current derivative curves with the applied voltage (Fig. 6(b)). Regarding sample S-SbN, the type-I band structure, which is just the addition of the two shown in Fig. 8, involves faster radiative recombination rates inside the $\mathrm{CL}$, further hindering carrier extraction.

\subsection{Alternative approaches for GaAsSbN CLS}

In an effort to obtain improved characteristics using the quaternary $\mathrm{CL}$, alternative structures containing simultaneously $\mathrm{Sb}$ and $\mathrm{N}$ were designed. Two new samples were fabricated following the same procedure described above for the first series of samples. The former structure consists of a $2.5 \mathrm{~nm}$-thick quaternary GaAsSbN CL (sample S-thin), in which a mitigation of carrier retrapping and a more efficient extraction from the thinner QW are expected. The latter one consists of a short-period superlattice $\mathrm{CL}$ containing 5 periods of $\mathrm{GaAsSb}(2 \mathrm{ML}) / \mathrm{GaAsN}(2 \mathrm{ML}$ ) (sample $\mathrm{S}-\mathrm{SL}$ ), which has been found to give rise to an improved structural quality of the CL as compared to the standard GaAsSbN alloy [39]. Both CLs were grown under the same conditions for the Sb and $\mathrm{N}$ sources, following previous growth optimization studies in single QD layers [26,39]. As Fig. 9(a) shows, these new structures lead to a significant enhancement of the $\mathrm{PC}$ at $0 \mathrm{~V}$ compared to the standard GaAsSbN CL, resulting in significant improvements in the $J_{\mathrm{sc}}$ of a $19 \%$ for S-SL and of a $49 \%$ for S-thin with a difference in $V_{\mathrm{oc}}$ within the $2 \%$ in both cases (not shown). The small variation in $V_{o c}$ in sample S-SL is in agreement with the similar expected $\mathrm{Sb}$ and $\mathrm{N}$ contents in the $\mathrm{CL}$. A reduction of the point defect density through the improved $\mathrm{CL}$ material could be partially responsible for the improvement in PC in this sample. Regarding sample S-thin, a more efficient extraction is now reflected in a much higher PC from the QDs and $\mathrm{CL}$, while the lower rate of carrier retrapping also leads to a slight enhancement of the PC originating from GaAs. However, the improved carrier extraction is not reflected in an enhanced $V_{o c}$, so that further investigations must be carried out to clarify this issue.

The $\mathrm{PC}$ at $-3 \mathrm{~V}$ reverse bias corresponding to these three samples are shown in Fig. 9(b) together with that from S-0 for comparison. As observed, at negative bias, all samples undergo significant PC enhancements. Indeed, in samples S-SL and S-thin, the PC contribution from GaAs becomes very close to that of sample S- 0 . Remarkably, the QD-CL-generated PC exceeds in these
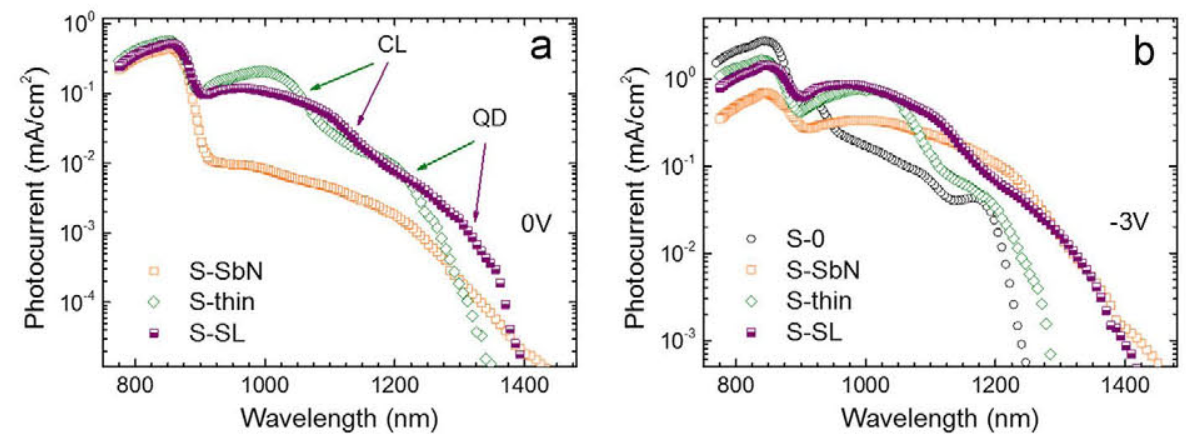

Fig. 9. (a) RT PC spectra from samples S-thin and S-SL together with that of S-SbN for a comparison. (b) RT PC from S-0, S-SbN, S-thin, and S-SL at -3 V. 
Table 1

Estimation of the integrated PC between 1.37 and $1.6 \mathrm{eV}$ ( $\mathrm{PC}_{\mathrm{GaAs}}$ ), related to the $\mathrm{GaAs}$ absorption, and for energies under $1.37 \mathrm{eV}\left(\mathrm{PC}_{\mathrm{QD}-\mathrm{CL}}\right)$, corresponding to the sub-bandgap (QD-QW) contribution.

\begin{tabular}{llllll}
\hline & \multicolumn{2}{l}{$\mathrm{PC}_{\mathrm{GaAs}}$} & & \multicolumn{2}{l}{$\mathrm{PC}$ (DD-CL } \\
\cline { 2 - 3 } \cline { 5 - 6 } & $0 \mathrm{~V}$ & $-3 \mathrm{~V}$ & & $0 \mathrm{~V}$ & $-3 \mathrm{~V}$ \\
\hline GaAs & 1 & 1 & - & - \\
S-0 & 0.94 & 1.14 & 0.13 & 0.21 \\
S-Sb & 1 & 1.13 & 0.31 & 0.38 \\
S-N & 0.26 & 1.04 & 0.15 & 0.65 \\
S-SbN & 0.17 & 0.29 & 0.01 & 0.35 \\
S-thin & 0.23 & 0.69 & 0.11 & 0.54 \\
S-SL & 0.21 & 0.63 & 0.11 & 0.73 \\
\hline
\end{tabular}

cases in a great extent the contribution of the sole presence of InAs QDs in standard GaAs-capped structure.

\subsection{Analysis of relative contributions to the total PC: impact of the CL nature}

In order to evaluate and compare the contribution of the $\mathrm{PC}$ from GaAs and from the QD-CL structure in all the samples, the integrated $\mathrm{PC}$ has been calculated for two different energy ranges at 0 and $-3 \mathrm{~V}$ applied biases. The estimations are summarized in table 1 , where $\mathrm{PC}_{\mathrm{GaAs}}$ represents the integrated $\mathrm{PC}$ for energies above the GaAs bandgap energy, between 1.37 and $1.6 \mathrm{eV}$, while $\mathrm{PC}_{\mathrm{QD}-\mathrm{CL}}$ corresponds to the integrated $\mathrm{PC}$ ascribed to the $\mathrm{QD}-\mathrm{CL}$ structure, for energies below $1.37 \mathrm{eV}$. Both values have been normalized to that of the control sample, which acts as reference, for a clear comparison among samples. At $0 \mathrm{~V}$, sample $\mathrm{S}$-Sb shows the highest integrated $\mathrm{PC}, \mathrm{PC}_{\mathrm{GaAs}}$ being similar to that from the control sample and $\mathrm{S}-0$, and $\mathrm{PC}_{\mathrm{QD}-\mathrm{CL}}$ being approximately 2.4 times higher than that in $\mathrm{S}-0$. In $\mathrm{N}$-containing samples, despite the strong reduction of $\mathrm{PC}_{\mathrm{GaAs}}, \mathrm{PC}_{\mathrm{QD}-\mathrm{CL}}$ from $\mathrm{S}-\mathrm{N}$ exceeds that from $\mathrm{S}-0$, while $\mathrm{PC}_{\mathrm{QD}-\mathrm{CL}}$ from S-thin and S-SL are comparable to it. At $-3 \mathrm{~V}$, all samples show increased integrated $P C$, with the exception of the control sample, as expected. This effect in $\mathrm{PC}_{\mathrm{GaAs}}$ and $\mathrm{PC} \mathrm{CD}_{\mathrm{QL}}$ from S-0 means that carrier loss mechanisms are also present in InAs/ GaAs structures with no CLs. This suggests that the InAs WL is thick enough to act as a trap for carriers, which could be the origin of the reduced $J_{\mathrm{sc}}$ compared to control sample and is in agreement with the shape of the IV curve under AM1.5 conditions. Remarkably, the fact that the relative increase at $-3 \mathrm{~V}$ is considerably smaller in sample $\mathrm{S}$-Sb indicates that the $\mathrm{GaAsSb} \mathrm{CL}$ is reducing the retrapping process and/or improving carrier extraction from the QDs (further investigations are ongoing to clarify this point). This leads to the measured enhancement in $J_{\mathrm{sc}}$. Regarding $\mathrm{N}$-containing samples, all of them undergo a very significant enhancement in PC under $-3 \mathrm{~V}$ reverse bias as compared to photovoltaic conditions. Particularly, an essentially complete carrier collection is now achieved in sample $\mathrm{S}-\mathrm{N}$, revealed from the value of $\mathrm{PC}_{\mathrm{GaAs}}$, approximately 1 . Although this value is not reached in the other $\mathrm{N}$-containing samples, all of them show a very significant increase not only in $\mathrm{PC}_{\mathrm{GaAs}}$, but also in $\mathrm{PC}_{\mathrm{QD}-\mathrm{CL}}$. The fact that $\mathrm{PC}_{\mathrm{GaAs}}$ approaches the saturation value at $-3 \mathrm{~V}$ and reaches it at around $-5 \mathrm{~V}$ (not shown) in samples S-thin and S-SL, reveals that extraction problems are now related to the type-I structure of the QWs rather than to the presence of defects. Therefore, this drawback could in principle be overcome by a stronger electric field in the intrinsic region. In addition, samples S-N, S-thin, and especially S-SL, show a $\mathrm{PC}_{\mathrm{OD}-\mathrm{CL}}$ much higher than $\mathrm{S}-0$ and $\mathrm{S}-\mathrm{Sb}$. The reduced accumulated strain in these structures as compared to $\mathrm{S}$-Sb could therefore be playing a positive role in the PC. Such $\mathrm{N}$-containing structures are therefore good candidates to achieve enhanced photoresponse and significantly exceed the value of $J_{s c}$ for standard GaAs solar cells if transport problems can be solved through an optimized solar cell design.

\section{Conclusions}

In conclusion, we have demonstrated the possibility to significantly extend the spectral photoresponse of InAs QD-based GaAs solar cells through the use of $\mathrm{GaAs}(\mathrm{Sb})(\mathrm{N}) \mathrm{CLs}$. The use of such CLs provides an additional contribution to $\mathrm{PC}$, making the devices act as hybrid QD-QW-GaAs multi-bandgap solar cells. The particular application of GaAsSb CLs leads to efficiencies significantly higher than those for a sample containing standard GaAs-capped QDs. However, the addition of $\mathrm{N}$ to the $\mathrm{CL}$ leads to a strong reduction in $J_{\mathrm{sc}}$, which is explained in terms of hindered electron extraction and retrapping in the N-containing QWs. Indeed, the $\mathrm{CL}$ potential depth, thickness, structure, and band alignment, have been found to play an important role in carrier extraction and transport, pointing to the fact that there is still room for optimized structures using GaAsSbN CLs leading to more efficient devices.

\section{Acknowledgments}

We acknowledge the Spanish MICINN-MINECO for funding through projects MAT2010-15206 and MAT2013-47102-C2-2-R. We would also like to thank Alicia Fraile (ISOM-UPM) for processing the samples.

\section{References}

[1] W. Shockley, H.J. Queisser, Detailed balance limit of efficiency of p-n junction solar cells, J. Appl. Phys. 32 (1961) 510.

[2] P.T. Landsberg, H. Nussbaumer, G. Willeke, Band-band impact ionization and solar cell efficiency, J. Appl. Phys. 74 (1993) 1451.

[3] A.J. Nozik, Spectroscopy and hot electron relaxation dynamics in semiconductor quantum wells and quantum dots, Annu. Rev. Phys. Chem. 52 (2001) 193.

[4] M.C. Beard, A.G. Midgett, M.C. Hanna, J.M. Luther, B.K. Hughes, A.J. Nozik, Comparing multiple exciton generation in quantum dots to impact ionization in bulk semiconductors: implications for enhancement of solar energy conversion, Nano Lett. 10 (2010) 3019.

[5] O.E. Semonin, J.M. Luther, S. Choi, H.-Y. Chen, J. Gao, A.J. Nozik, M.C. Beard, Peak external photocurrent quantum efficiency exceeding $100 \%$ via MEG in a quantum dot solar cell, Science 334 (2011) 1530.

[6] A. Luque, A. Marti, Increasing the efficiency of ideal solar cells by photon induced transitions at intermediate levels, Phys. Rev. Lett. 78 (1997) 5014.

[7] D. Guimard, R. Morihara, D. Bordel, K. Tanabe, Y. Wakayama, M. Nishioka, Y. Arakawa, Fabrication of $\ln A$ s/GaAs quantum dot solar cells with enhanced photocurrent and without degradation of open circuit voltage, Appl. Phys. Lett. 96 (2010) 203507.

[8] C.G. Bailey, D.V. Forbes, R.P. Raffaelle, S.M. Hubbard, Near 1 V open circuit voltage InAs/GaAs quantum dot solar cells, Appl. Phys. Lett. 98 (2011) 163105.

[9] W.S. Liu, H.M. Wu, F.H. Tsao, T.L. Hsu, J.-I. Chyi, Improving the characteristics of intermediate-band solar cell devices using a vertically aligned $\ln \mathrm{As} / \mathrm{GaAsSb}$ quantum dot structure, Sol. Energy Mater. Sol. Cells 105 (2012) 237.

[10] S.M. Hubbard, A. Podell, C. Mackos, S. Polly, C.G. Bailey, D.V. Forbes, Effect of vicinal substrates on the growth and device performance of quantum dot solar cells, Sol. Energy Mater. Sol. Cells 108 (2013) 256.

[11] A. Sayari, M. Ezzidini, B. Azeza, S. Rekaya, E. Shalaan, S.J. Yaghmour, A.A. Al-Ghamdi, L. Sfaxi, R. M'ghaieth, H. Maaref, Improvement of performance of GaAs solar cells by inserting self-organized $\ln A s / \ln \mathrm{naAs}$ quantum dot superlattices, Sol. Energy Mater. Sol. Cells 113 (2013) 1.

[12] J.M. Ulloa, R. Gargallo-Caballero, M. Bozkurt, M. del Moral, A. Guzman, P.M. Koenraad, A. Hierro, GaAsSb-capped InAs quantum dots: from enlarged quantum dot height to alloy fluctuations, Phys. Rev. B 81 (2010) 165305.

[13] R. Teissier, D. Sicault, J. Harmand, G. Ungaro, G. Le Roux, L. Largeau, Temperature-dependent valence band offset and bandgap energies of pseudomorphic GaAsSb on GaAs, J. Appl. Phys. 89 (2001) 5473.

[14] M.M. Bajo, J.M. Ulloa, M. Del Moral, A. Guzmán, A. Hierro, Near infrared InAs/ GaAsSb quantum dot light emitting diodes, IEEE J. Quantum Electron. 47 (2011) 1547. 
[15] A.D. Utrilla, J.M. Ulloa, A. Guzman, A. Hierro, Impact of the Sb content on the performance of GaAsSb-capped InAs/GaAs quantum dot lasers, Appl. Phys. Lett 103 (2013) 111114

[16] C.T. Huang, Y.C. Chen, S.C. Lee, Improved photoresponse of InAs/GaAs quantum dot infrared photodetectors by using $\mathrm{GaAs}_{1-x} \mathrm{Sb}_{x}$ strain reducing layer, Appl. Phys. Lett. 100 (2012) 043512

[17] X.Q. Zhang, S. Ganapathy, I. Suemune, H. Kumano, K. Uesugi, Improvement of InAs quantum-dot optical properties by strain compensation with GaNAs capping layers, Appl. Phys. Lett. 83 (2003) 4524.

[18] O. Schumann, S. Birner, M. Baudach, L. Geelhaar, H. Eisele, L. Ivanova, R. Timm, A. Lenz, S.K. Becker, M. Povolotskyi, M. Dähne, G. Abstreiter, H. Riechert, Effects of strain and confinement on the emission wavelength of InAs quantum dots due to a $\mathrm{GaAs}_{1-x} \mathrm{~N}_{x}$ capping layer, Phys. Rev. B 71 (2005) 245316.

[19] W. Shan, W. Walukiewicz, J.W. Ager III, E.E. Haller, J.F. Geisz, D.J. Friedman, J.M. Olson, S.R. Kurtz, Band anticrossing in GaInNAs alloys, Phys. Rev. Lett. 82 (1999) 1221.

[20] R. Oshima, A. Takata, Y. Okada, Strain-compensated InAs/GaNAs quantum dots for use in high efficiency solar cells, Appl. Phys. Let 93 (2008) 083111.

[21] J.M. Ulloa, D.F. Reyes, M. Montes, K. Yamamoto, D.L. Sales, D. González, Independent tuning of electron and hole confinement in InAs/GaAs quantum dots through a thin GaAsSbN capping layer, Appl. Phys. Lett. 100 (2012) 013107

[22] R.B. Laghumavarapu, M. El-Emawy, N. Nuntawong, A. Moscho, L.F. Lester, D.L. Huffaker, Improved device performance of $\ln A s /$ GaAs quantum dot solar cells with GaP strain compensation layers, Appl. Phys. Lett. 91 (2007) 243115.

[23] S.M. Hubbard, C.D. Cress, C.G. Bailey, R.P. Raffaelle, S.G. Bailey, D.M. Wilt, Effect of strain compensation on quantum dot enhanced GaAs solar cells, Appl. Phys. Lett. 92 (2008) 123512.

[24] V. Popescu, G. Bester, M.C. Hanna, A.G. Norman, A. Zunger, Theoretical and experimental examination of the intermediate-band concept for strainbalanced (In,Ga)As/Ga(As,P) quantum dot solar cells, Phys. Rev. B 78 (2008) 205321.

[25] N.S. Beattie, G. Zoppi, P. See, 1. Farrer, M. Duchamp, D.J. Morrison, R.W. Miles, D.A. Ritchie, Analysis of InAs/GaAs quantum dot solar cells using Suns- $V_{\text {or }}$ measurements, Sol. Energy Mater. Sol. Cells 130 (2014) 241.

[26] A.D. Utrilla, J.M. Ulloa, A. Guzman, A. Hierro, Long-wavelength room-temperature luminescence from $\ln A s /$ GaAs quantum dots with an optimized GaAsSbN capping layer, Nanoscale Res. Lett. 9 (2014) 36.

[27] J.M. Ulloa, D.F. Reyes, A.D. Utrilla, A. Guzman, A. Hierro, T. Ben, D. González, Capping layer growth rate and the optical and structural properties of
GaAsSbN-capped InAs/GaAs quantum dots, Appl. Phys. Lett. 116 (2014) 134301.

[28] P.G. Linares, E. López, I. Ramiro, A. Datas, E. Antolín, Y. Shoji, T. Sogabe, Y. Okada, A. Martí, A. Luque, Voltage limitation analysis in strain-balanced InAs/GaAsN quantum dot solar cells applied to the intermediate band concept, Sol. Energy Mater. Sol. Cells 132 (2015) 178.

[29] S. Wicaksono, S.F. Yoon, K.H. Tan, W.K. Cheah, Concomitant incorporation of antimony and nitrogen in GaAsSbN lattice-matched to GaAs, J. Cryst. Growth 274 (2005) 355.

[30] A. Alemu, J.A.H. Coaquira, A. Freundlich, Dependence of device performance on carrier escape sequence in multi-quantum-well $\mathrm{p}-\mathrm{i}-\mathrm{n}$ solar cells, J. Appl. Phys. 99 (2006) 084506.

[31] B.Q. Sun, D.S. Jiang, X.D. Luo, Z.Y. Xu, Z. Pan, L.H. Li, R.H. Wu, Interband luminescence and absorption of GaNAs/GaAs single-quantum-well structures, Appl. Phys. Lett. 76 (2000) 2862.

[32] H. Fujii, Y. Wang, K. Watanabe, M. Sugiyama, Y. Nakano, High-aspect-ratio structures for efficient light absorption and carrier transport in InGaAs/GaAsP multiple quantum well solar cells, IEEE J. Photovolt. 3 (2013) 859.

[33] 1. Serdiukova, C. Monier, M.F. Vilela, A. Freundlich, Critical built-in electric field for an optimum carrier collection in multiquantum well p-i-n diodes, Appl. Phys. Lett. 74 (1999) 2812.

[34] H.G. Khalil, N. Balkan, Carrier trapping and escape time in p-i-n GaInNAs MoW structures, Nanoscale Res. Lett. 9 (2014) 21.

[35] B. Royall, H. Khalil, S. Mazzucato, A. Erol, N. Balkan, Experimental investigation and numerical modelling of photocurrent oscillations in lattice matched $\mathrm{Ga}_{1-x} \mathrm{In}_{x} \mathrm{~N}_{y} \mathrm{As}_{1-y} / \mathrm{GaAs}$ quantum well $\mathrm{p}-\mathrm{i}-\mathrm{n}$ photodiodes, Nanoscale Res. Lett. 9 (2014) 84.

[36] U. Bockelmann, T. Egeler, Electron relaxation in quantum dots by means of Auger processes, Phys. Rev. B 46 (1992) 15574.

[37] J.M. Ulloa, J.L. Sánchez-Rojas, A. Hierro, J.M.G. Tijero, E. Tournié, Effect of nitrogen on the band structure and material gain of $\ln _{y} \mathrm{Ga}_{1-y} \mathrm{As}_{1-x} \mathrm{~N}_{x}-\mathrm{GaAs}$ quantum wells, IEEE J. Sel. Top. Quantum Electron. 9 (2003) 716.

[38] H. Schneider, K.V. Klitzing, Thermionic emission and gaussian transport of holes in a GaAs/ $/ \mathrm{Al}_{\times} \mathrm{Ga}_{1-\chi}$ As multiple-quantum-well structure, Phys. Rev. B 38 (1988) 6160.

[39] A.D. Utrilla, D.F. Reyes, J.M. Ulloa, D. González, T. Ben, A. Guzman, A. Hierro, GaAsSb/GaAsN short-period superlattices as a capping layer for improved InAs quantum dot-based optoelectronics, Appl. Phys. Lett. 105 (2014) 043105. 